\title{
Identifying Trojan Facebook Applications
}

\author{
Gowtham Kumar Pullagujju1)
}

\begin{abstract}
With 20 million introduces a day, third-party applications are a noteworthy purpose behind the fame and addictiveness of Facebook. Shockingly, programmers have understood the capability of utilizing applications for spreading malware and spam. The issue is as of now huge, as we find that no less than $13 \%$ of applications in our dataset are noxious. Up until this point, the examination group has concentrated on distinguishing vindictive posts and battles. In this paper, we ask the question: Given a Facebook application, would we be able to figure out whether it is malignant? Our key commitment is in creating FRAppE-Facebook's Rigorous Application Evaluator-ostensibly the principal device concentrated on recognizing vindictive applications on Facebook. To create FRAppE, we utilize data assembled by watching the posting conduct of $111 \mathrm{~K}$ Facebook applications seen crosswise over 2.2 million clients on Facebook. Initially, we recognize an arrangement of elements that help us recognize malignant applications from favorable ones. For instance, we find that vindictive applications frequently impart names to different applications, and they regularly ask for less authorizations than kindhearted applications. Second, utilizing these recognizing highlights, we demonstrate that FRAppE can distinguish malignant applications with 99.5\% exactness, with no false positives and a high genuine positive rate $(95.9 \%)$. At long last, we investigate the biological community of malevolent Facebook applications and recognize instruments that these applications use to proliferate. Strangely, we find that numerous applications conspire and bolster each other; in our dataset, we find 1584 applications empowering the viral proliferation of 3723 different applications through their posts. Long haul, we consider FRAppE to be a stage toward making an autonomous guard dog for application evaluation and positioning, in order to caution Facebook users before installing apps.
\end{abstract}

Keywords : facebook apps, malicious, online social networks, spam, malware.

\section{Introduction}

Online social networks (OSNs) empower and support third-party applications (apps) to improve the client encounter on these stages. Such upgrades incorporate fascinating or engaging methods for imparting among online companions and different exercises, for example, playing recreations or tuning in to tunes. For instance, Facebook gives designers an API [1][2] that encourages application incorporation into the Facebook client encounter. There are 500K

Received(August 5, 2016), Review Result(1st: August 25, 2016, 2nd: September 23, 2016), Accepted(December 10, 2016)

${ }^{1}$ (Corresponding Author) Department of Computer Science and Engineering, KL University

email: pullagujjugowtham@gmail.com 
applications accessible on Facebook[3], and by and large, 20M applications are introduced each day [1]. Moreover, numerous applications have procured and keep up a truly expansive client base. For example, FarmVille and CityVille applications have $26.5 \mathrm{M}$ and $42.8 \mathrm{M}$ clients to date.

As of late, programmers have begun exploiting the ubiquity of this outsider applications stage and deploying malicious applications [4-6]. Malicious applications can give a lucrative business to programmers, given the prominence of OSNs, with Facebook driving the path with 900M dynamic clients [7].

There are numerous ways that programmers can profit by a vindictive application:

1) the application can achieve huge quantities of clients and their companions to spread spam;

2) the application can acquire clients' close to home data, for example, email address, main residence, and sexual orientation; and

3) the application can "replicate" by making different pernicious applications well known.

To exacerbate matters, the arrangement of vindictive applications is improved by prepared to-utilize toolboxs beginning at $\$ 25$ [8]. At the end of the day, there is thought process and opportunity, and subsequently, there are numerous malignant applications spreading on Facebook consistently [9][10].

In spite of the above troubling patterns, today a client has extremely constrained data at the season of introducing an application on Facebook. As it were, the issue is the accompanying: Given an application's character number (the one of a kind identifier doled out to the application by Facebook), would we be able to recognize if the application is noxious? Right now, there is no business benefit, openly accessible data, or research-based apparatus to educate a client about the dangers with respect to an application.

\section{Proposed system}

\subsection{Related works}

Detecting Spam on OSNs: Gao et al. [11] examined posts on the dividers of 3.5 million Facebook clients and demonstrated that $10 \%$ of connections posted on Facebook dividers are spam. They likewise exhibited strategies to distinguish traded off records and spam battles. In other work, Gao et al. [12] and Rahman et al. [10] created productive procedures for online 
spam sifting on OSNs, for example, Facebook. While Gao et al. [12] depended on having the entire social chart as information, as is usable just by the OSN supplier, Rahman et al. [10] built up an outsider application for spam discovery on Facebook. Others [11] and [12] showed systems for discovery of spam URLs on Twitter. As opposed to these endeavors, as opposed to grouping singular URLs or posts as spam, we concentrate on distinguishing malicious applications that are the main source of spam on Facebook.

Application Permission Exploitation: Chia et al. [13] investigated risk signaling on the protection meddling of Facebook applications and reason that ebb and flow types of group appraisals were not solid pointers of the security dangers related with an application. Additionally, with regards to our perception, they found that mainstream Facebook applications tend to demand more authorizations.

\subsection{Existing system}

- So far, the exploration group has given careful consideration to OSN applications particularly. Most research identified with spam and malware on Facebook has concentrated on distinguishing malevolent posts and social spam campaigns.

- Gao et al. [11] investigated posts on the dividers of 3.5 million Facebook clients and demonstrated that $10 \%$ of connections posted on Facebook dividers are spam. They additionally displayed systems to recognize compromised accounts and spam campaigns.

- Yang et al. [18] and Benevenuto et al. [19] created strategies to recognize records of spammers on Twitter. Others have proposed a honey-pot-based approach to detect spam accounts on OSNs.

- Chia et al.[13] investigate chance motioning on the security nosiness of Facebook applications and infer that present types of group evaluations are not dependable markers of the protection dangers related with an application.

\subsection{Disadvantages of exiting system}

Existing system works focused just on ordering singular URLs or posts as spam, yet not concentrated on recognizing malicious applications that are the fundamental source of spam on Facebook.

Existing system works concentrated on records made by spammers rather than malicious 
application.

Existing system gave just an abnormal state outline about threats to the Facebook graph and don't give any examination of the system.

\subsection{Proposed system}

In this paper, we create FRAppE, a suite of productive order strategies for distinguishing whether an application is malignant or not. To assemble FRAppE, we utilize information from MyPage-Keeper, a security application in Facebook.

We find that malicious applications essentially vary from kindhearted applications regarding two classes of components: On-Demand Features and Aggregation-Based Features.

We exhibit two variations of our pernicious application classifier-FRAppE Lite and FRAppE.

FRAppE Lite is a lightweight form that makes utilization of just the application highlights accessible on request. Given a particular application ID, FRAppE Lite slithers the on-request includes for that application and assesses the application in light of these components progressively.

FRAppE-a malicious application detector that uses our aggregation-based elements notwithstanding the on-demand features.

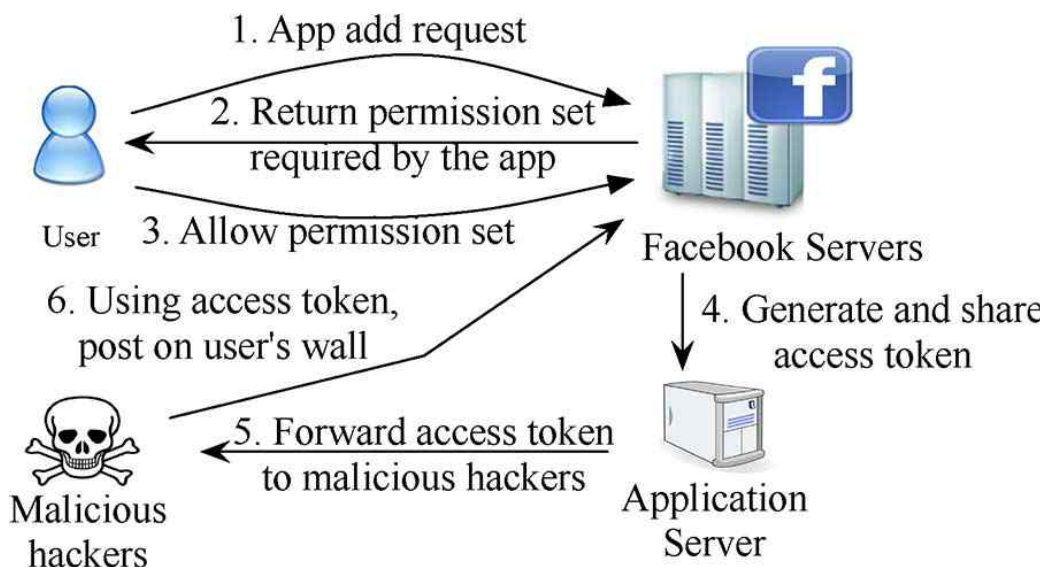

[Fig. 1] System model

\subsection{Advantage of proposed system}

- The proposed work is seemingly the primary extensive review concentrating on malignant 
Facebook applications that spotlights on evaluating, profiling, and comprehension vindictive applications and incorporates this data into a compelling recognition approach.

- Several highlights utilized by FRAppE, for example, the notoriety of divert URIs, the quantity of required consents, and the utilization of various customer IDs in application establishment URLs, are powerful to the development of programmers.

- Not utilizing distinctive customer IDs in application installation URLs would restrict the capacity of programmers to instrument their applications to proliferate each other.

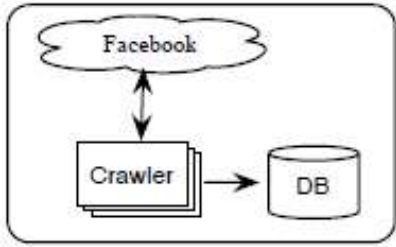

Crawling Facebook post:

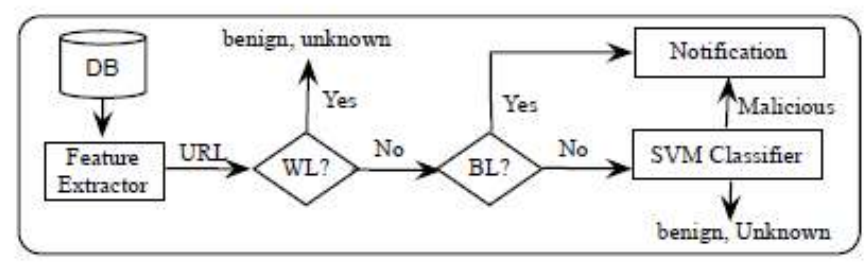

Classifying Facebook posts

[Fig. 2] System architecture

\section{Conclusion}

Applications show helpful means for programmers to spread pernicious substance on Facebook. Notwithstanding, little is comprehended about the qualities of malignant applications and how they work. In this paper, utilizing a substantial corpus of pernicious Facebook applications saw over a 9-month time frame, we demonstrated that noxious applications contrast fundamentally from generous applications regarding a few elements. For instance, vindictive applications are substantially more liable to impart names to different applications, and they regularly ask for less consents than kind applications. Utilizing our perceptions, we created FRAppE, a precise classifier for recognizing vindictive Facebook applications. Most curiously, we highlighted the rise of application nets-substantial gatherings of firmly associated applications that advance each other. We will keep on digging further into this biological community of pernicious applications on Facebook, and we trust that Facebook will profit by our proposals for diminishing the hazard of programmers on their platform.

\section{References}

[1] C. Pring, 100 social media statistics for 2012, (2012) [Online]. Available: 
http://thesocialskinny.com/100-social-media-statistics-for-2012/

[2] Facebook, Palo Alto, CA, USA, Facebook Opengraph API, [Online]. Available: http://developers.facebook.com/docs/reference/api/

[3] Wiki: Facebook platform, (2014) [Online]. Available: http://en.wikipedia.org/wiki/Facebook_Platform

[4] Profile stalker: Rogue Facebook application, (2012) [Online]. Available: https://apps.facebook.com/mypagekeeper/?status=scam_report-_fb_survey_scam_profile_viewer_2012_4_4

[5] Whiich cartoon character are you-Facebook surveyscam, (2012) [Online]. Available: https://apps.facebook.com/mypagekeeper/?status=scam_report_fb_survey_scam_whiich_cartoon_character_are_you_ 2012_03_30

[6] G. Cluley, The Pink Facebook rogue application and survey scam, (2012) [Online]. Available: http://nakedsecurity.sophos.com/2012/02/27/pink-facebook-survey-scam/

[7] D. Goldman, Facebook tops 900 million users, (2012) [Online]. Available: http://money.cnn.com/2012/04/23/technology/facebookq1/index.htm

[8] R. Naraine, Hackers selling \$25 toolkit to create malicious Facebookapps, (2011) [Online]. Available: http://zd.net/g28HxI

[9] HackTrix, Stay away from malicious Facebook apps, (2013) [Online]. Available: http://bit.ly/b6gWn5

[10] M. S. Rahman, T. K. .Huang, H. V. Madhyastha, and M. Faloutsos, Efficient and scalable socware detection in online social networks, Proc. USENIX Security, (2012), p.32.

[11] H. Gao, J. Hu, C. Wilson, Z. Li, Y. Chen, and B. Y. Zhao., Detecting and characterizing social spam campaigns, Proc. IMC, (2010), pp.35-47.

[12] H. Gao, Y. Chen, K. Lee, D. Palsetia, and A. Choudhary, Towardsonline spam filtering in social networks, Proc. NDSS, (2012).

[13] P. Chia, Y. Yamamoto, and N. Asokan, Is this app safe? A large scale study on application permissions and risk signals, Proc. WWW, (2012), pp.311-320.

[14] WhatApp? (beta)-A Stanford Center for Internet and SocietyWebsite with support from the Rose Foundation, [Online]. Available: https://whatapp.org/facebook/

[15] MyPageKeeper, [Online]. Available: https://www.facebook.com/apps/application.php?id=167087893342260

[16] Facebook, Palo Alto, CA, USA, Facebook platform policies, [Online]. Available: https://developers.facebook.com/policy/

[17] Facebook, Palo Alto, CA, USA, Application authentication flow using OAuth 2.0, [Online]. Available: http://developers.facebook.com/docs/authentication/

[18] C. Yang, R. Harkreader, and G. Gu, Die free or live hard? Empirical evaluation and new design for fighting evolving Twitter spammers, Proc. RAID, (2011), pp.318-337.

[19] F. Benevenuto, G. Magno, T. Rodrigues, and V. Almeida, Detectingspammers on Twitter, Proc. CEAS, (2010), pp.1-9. 
[20] G. Stringhini, C. Kruegel, and G. Vigna, Detecting spammers on socialnetworks, Proc. ACSAC, (2010), pp.1-9. 
Identifying Trojan Facebook Applications

(This page is empty intentionally) 